\title{
Dopamine treatment for severe ovarian hyperstimulation syndrome
}

\author{
A.P.Ferraretti ${ }^{1.4}$, L.Gianaroli ${ }^{2}$, L.Diotallevi ${ }^{1}$, \\ C.Festi ${ }^{1}$ and A.Trounson ${ }^{3}$ \\ 'Villa Regina, IVF Centre, Via Castıglione, 115, 40136 Bologna, \\ Italy, ${ }^{2}$ Clinique de Stérnlité et d'Endocrinologie Gynécologique, \\ Université de Genève, 32, Boulevard de le Cluse, Genève 1211, \\ Switzerland and ${ }^{3}$ Centre for Early Human Development, Monash \\ Medical Centre, 246 Clayton Road, Clayton, Victoria 3168 , \\ Australia \\ ${ }^{4}$ To whom correspondence should be addressed
}

Seven oliguric patients with severe ovarian hyperstimulation syndrome following gonadotrophin treatment for in-vitro fertilization or gamete intra-Fallopian transfer, were treated with low doses of dopamine by peripheral infusion. Five patients were pregnant. The rationale for this therapeutic approach was to increase renal blood flow and glomerular filtration. In addition to dopamine, fluid intake was restricted to $500 \mathrm{ml} /$ day and a protein and salt-rich diet was provided in order to increase serum osmolarity. Within $24-48 \mathrm{~h}$ from the beginning of the dopamine treatment, the syndrome started to regress in all cases. No adverse maternal or fetal effects occurred. We conclude that dopamine therapy may constitute a major advance towards the management of severe ovarian hyperstimulation syndrome.

Key words: dopamine/ovarian hyperstimulation syndrome

\section{Introduction}

Ovarian hyperstimulation syndrome (OHS) is a well recognized iatrogenic complication of gonadotrophin therapy during treatment for infertility. Increased hormonal monitoring and ovarian ultrasound examination have reduced the incidence and the severity of OHS during ovarian stimulation for the induction of ovulation for amenorthoea. However, OHS cannot be completely avoided because of the narrow margin which exists between the successful induction of ovulation and the sudden growth of multiple follicles leading to OHS. In addition, there has been a rapid increase in the number of women treated by in-vitro fertilization (IVF) and associated techniques, such as gamete intraFallopian transfer (GIFT), with gonadotrophins which stimulate the production of multiple follicles and oocytes, thus increasing the likelihood of OHS in these patients. In our own infertility programme, which has involved 1117 IVF and GIFT treatment cycles, seven patients $(0.6 \%)$ have experienced severe OHS.

A variety of treatments for OHS have been proposed but because little is known about the physiology of the syndrome, there is no general agreement on the management of these patients. In the present paper, we report the results of a new therapeutic approach based on the peripheral infusion of low doses of dopamine. In OHS patients, there is a rapid and massive fluid shift from the intravascular compartment into the peritoneal and pleural cavities due to increased capillary permeability (Haning et al., 1985; Navot et al., 1987). The resulting hypovolaemia leads to decreased renal perfusion and secondarily to increased salt and water reabsorption in the proximal renal tubules. The reabsorbed fluid and sodium are transferred rapidly to the 'third space' and the patient remains hypovolaemic while ascites further increases. A prolonged hypovolaemia can cause vasoconstriction of afferent arterioles producing kidney failure as effective perfusion of kidney parenchyma decreases.

Dopamine produces its renal effect by increasing renal blood flow and glomerular filtration. This is accomplished via stimulation of the dopaminergic receptors present in the vascular kidney bed (Felder et al. , 1984). The rationale for treating oliguric OHS patients with dopamine is to avoid fluid and salt retention and to prevent acute renal failure.

\section{Materials and methods}

Seven patients with severe OHS were admitted to hospital after induction of multiple follicular growth using follicle stimulating hormone (FSH, Metrodin; Serono Laboratories, Rome, Italy) and human menopausal gonadotrophin (HMG, Pergonal; Serono Laboratories, Rome, Italy), for IVF or GIFT as described elsewhere (Gianaroli et al., 1986). In two cases, the gonadotrophin-releasing hormone $(\mathrm{GnRH})$ analogue buserelin (Suprefact; Hoechst UK Ltd) was administered subcutaneously 3 weeks before and during ovarian stimulation. All patients were given an ovulatory dose of 5000 or 10000 IU human chorionic gonadotrophin (HCG, Profasi; Serono Laboratories) $34 \mathrm{~h}$ prior to oocyte recovery, and all received progesterone supplementation throughout the luteal phase $(50 \mathrm{mg}$ i.m./day) (Gestone; AMSA Lab., Florence, Italy).

Abdominal discomfort which required hospitalization arose 7 and 6 days after HCG in the two non-pregnant women. The other five patients, all pregnant, were admitted 15 to 18 days after HCG. At the time of hospitalization all patients had greatly enlarged ovaries, easily discernible by abdominal palpation, and marked ascites. In five patients there was associated pleural effusion. The clinical manifestation of this involved oliguria, signs of hypovolaemia, weight gain and respiratory distress. Laboratory tests showed an imbalance of electrolytes (low urinary excretion), loss of serum total protein and slightly increased fibrinogen in all cases.

In five cases, serum osmolarity was low. In five cases the 
Table I. Clinical and laboratory parameters at the tume of hospitalization of patients suffering from severe ovarian hyperstimulation syndrome

\begin{tabular}{|c|c|c|c|c|c|c|c|c|}
\hline Patient number & Normal range & 1 & 2 & 3 & 4 & 5 & 6 & 7 \\
\hline Hydrothorax & - & Yes & Yes & Yes & No & Yes & No & Yes \\
\hline Diuresis $(\mathrm{ml} / 24 \mathrm{~h})$ & $(1000-1500)$ & 130 & 250 & 500 & 300 & 400 & 280 & 300 \\
\hline Pulse frequency/min & $(60-80)$ & 90 & 96 & 96 & 100 & 96 & 110 & 80 \\
\hline Ovarian volume $(\mathrm{ml})$ Right/Left & $(100-200)$ & $374 / 175$ & $314 / 332$ & $792 / 770$ & $762 / 616$ & $636 / 512$ & $361 / 332$ & $251 / 380$ \\
\hline Haematocnt ( $\%$ ) & $(37-42)$ & 43 & 35 & 43 & 47 & 45 & 48 & 34 \\
\hline Serum creatinine (mg $\%$ ) & $(0.5-1.2)$ & 1.23 & 1.2 & 098 & 1.35 & 1.3 & 1.21 & 0.81 \\
\hline Serum $\mathrm{Na}(\mathrm{mEq} / \mathrm{l})$ & $(132-145)$ & 138 & 138 & 135 & 139 & 135 & 140 & 139 \\
\hline Serum $K(m E q / l)$ & $(4-45)$ & 4.6 & 4.2 & 3.9 & 4.2 & 4.1 & 4.9 & 4.2 \\
\hline Serum osmolality $(\mathrm{mOsm} / \mathrm{kg}$ ) & $(278-305)$ & 290 & 269 & 279 & 277 & 267 & 279 & 290 \\
\hline Unnary $\mathrm{Na}(\mathrm{mEq} / 24 \mathrm{~h})$ & $(130-220)$ & 93 & 45 & 85 & 37 & 69 & 53 & 19 \\
\hline Urinary K (mEq/24 h) & $(50-100)$ & 70 & 23 & 58 & 29 & 44 & 40 & 18 \\
\hline
\end{tabular}

haematocrit value increased $>6-10 \%$, indicating haemoconcentration. In five cases, serum creatinine was above or at the upper limit of the normal range, indicating inadequate renal function due to decreased perfusion. Blood urea was in the normal range.

Management of the patients consisted of bed rest, restriction of fluid intake to $500 \mathrm{ml} /$ day, daily monitoring of urine output, weight, abdominal girth and haematological, biochemical and clotting parameters, as well as serial measurements of blood pressure and pulse rate. In the pregnant women, a $\beta$-subunit for HCG assay was performed every 2 days. The patients were given a protein- and salt-rich diet in order to increase oncotic and osmotic blood pressure. An ultrasound pelvic scan was carried out every $3-5$ days.

Dopamine treatment commenced within $10 \mathrm{~h}$ of admittance to hospital using an intravenous infusion pump (Terufusion, STC/502; Terumo Corp., Tokyo, Japan) which strictly controlled the dosage of dopamine delivered. The dopamine dosage used was $4.32 \mathrm{mg} / \mathrm{kg}$ body weight $/ 24 \mathrm{~h}$. This dose was capable of dilating renal vessels and increasing renal blood flow without affecting blood pressure and pulse rate (Kirshan et al., 1988). Dopamine treatment was continued until there was a complete resolution of ascites. Plasma expanders or albumin were only administered if required. Anticoagulants to prevent thromboembolic phenomena were given if clotting factors were altered.

\section{Results}

The clinical details and urinary and blood parameters of patients when admitted to hospital are shown in Table I.

In three cases, the first ultrasound examination revealed a multiple implantation. One patient (no. 2) had twin gestational sacs in the uterus and one in the left tube requiring salpingectomy at laparotomy; nos. 6 and 7 had intrauterine triplets and twins respectively.

In the two non-pregnant women, dopamine treatment was required for only 7 days. In the pregnant women, dopamine treatment was required for $9-22$ days. The duration of treatment was related to the magnitude of the increase of endogenous HCG and was longest in those patients with triplets (18-22 days), shortest in those women with a single fetus (9-10 days) and

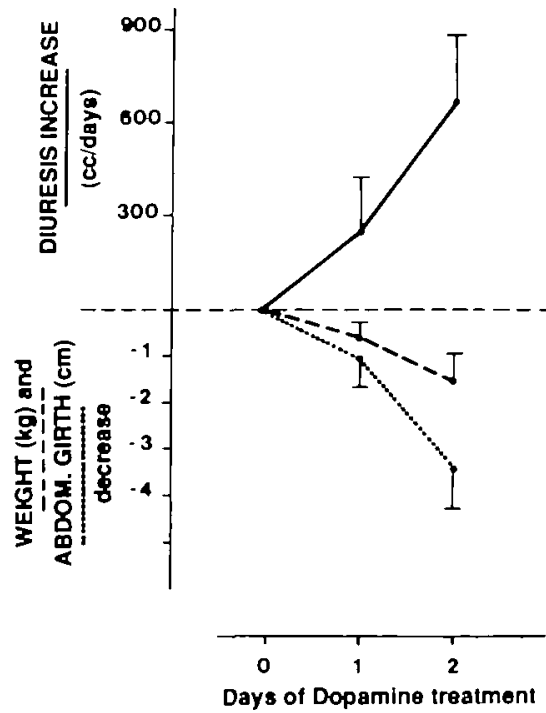

Fig. 1. Variation of diuresis (--), weight (--) and abdomınal girth $(\ldots . .$.$) in the first 48 \mathrm{~h}$ of dopamine treatment in seven patients suffering from severe ovarian hyperstimulation syndrome (Mean $\pm S D$ ).

intermediate for those with twins (14 days). Despite restriction of fluid intake to $500 \mathrm{ml} /$ day, all patients showed a considerable increase in diuresis and a concomitant decrease in weight and abdominal girth within 24 to $48 \mathrm{~h}$ of dopamine treatment (Figure 1). Urinary sodium and potassium excretion returned rapidly to the normal range (Figure 2). When haemoconcentration was present at the time of hospitalization, the serial determination of haematocrit under treatment showed a rapid decrease in all cases and it was stabilized between 35 and $40 \%$ by the fourth day of treatment. Other altered biochemical parameters required varying periods of time to return to normal: $3-7$ days for total plasma proteins and 4-8 days for serum creatinine. These urinary and blood parameters then remained within the normal range during the remainder of the treatment period.

Plasma expanders $(500 \mathrm{ml})$ were infused into patient 2 on the second and fourth days after laparotomy because of severe hypotension. The classical treatment of infusing albumin to 
Table II. Variatıons of clinical and laboratory parameters between the onset and end of dopamıne treatment in patients suffenng from severe ovanan hypersımulation treatment

\begin{tabular}{|c|c|c|c|c|c|c|c|}
\hline Patient number & 1 & 2 & 3 & 4 & 5 & 6 & 7 \\
\hline \multicolumn{8}{|l|}{ Clinical signs } \\
\hline Weight (kg) & -39 & -8.4 & -8.1 & -3.1 & -2 & -5.5 & -14 \\
\hline Abdominal girth $(\mathrm{cm})$ & -7 & -5 & -13 & -7 & -11 & 12 & -6 \\
\hline Diuresıs $(\mathrm{ml} / 24 \mathrm{~h})$ & +1270 & +750 & +700 & +700 & +1400 & +520 & +400 \\
\hline Pulse frequency/min & -18 & -12 & -14 & -32 & -16 & -22 & 0 \\
\hline \multicolumn{8}{|l|}{ Ovarian volume (ml) } \\
\hline Right ovary & -182 & -280 & -141 & -370 & -220 & +150 & -116 \\
\hline Left ovary & -80 & -320 & -159 & -322 & -150 & +140 & -134 \\
\hline \multicolumn{8}{|l|}{ Laboratory data } \\
\hline Serum protein ( $\mathrm{g}$ ) & +1.4 & +0.5 & +12 & +14 & +1.2 & +16 & +02 \\
\hline Serum creatinne (mg $\%$ ) & -0.33 & -0.1 & -03 & -0.53 & -04 & 0 & 0 \\
\hline Fibrinogen (mg $\%)$ & -330 & -64 & -75 & -264 & -127 & -63 & -40 \\
\hline Haematocrit (\%) & -3 & -1 & -6 & -11 & -6 & 15 & 0 \\
\hline
\end{tabular}

increase oncotic blood pressure was only needed in two of the patients (nos. 1 and 2) in a very low dose (a total of 15 and $10 \mathrm{~g}$ respectively). Despite high fibrinogen levels in all cases, we only treated patients with heparin if alteration of other clotting parameters was observed. It was known that elevation of plasma fibrinogen is often associated with ascites without necessarily indicating blood hypercoagulability.

During the monitoring period, two patients showed a mild alteration in liver function tests [increased serum aspartate aminotransferase (SGOT), serum alanine aminotransferase (SGPT) and $\gamma$-glutamyl transpeptidase $(\gamma$-GT)] as reported by others (Younis et al., 1988; Ryley et al., 1990).

Dopamine infusion was stopped only when an ultrasonic examination displayed a complete reabsorption of the peritoneal fluid. At that time, all the parameters considered were within the normal range. The ovaries were still enlarged in all subjects at the completion of dopamine infusion. It was well known (Schenker and Weinstein, 1978) that ovaries of OHS patients require more time to return to their normal size than it takes for the clinical syndrome to regress, particularly when endogenous HCG stimulation of ovaries has occurred. No further complications were observed after discontinuation of dopamine in any of the women.

In Table II, the progression of the values of the clinical and laboratory tests between the start and finish of treatment are shown.

To date, five pregnant patients have delivered seven healthy babies. Patient nos. 6 and 2 spontaneously aborted one of the intrauterine gestational sacs in the first trimester, carrying to term normal twins and a singleton respectively.

\section{Discussion}

All treatments proposed in the literature for severe OHS are to some extent controversial. Intravenous administration of volume expanders to correct hypovolaemia (Schenker and Weinstein, 1978) can increase intravascular volume only transiently and can cause a further increase of interstitial fluid accumulation. This must only be used as an emergency measure in the correction of frank hypotension or renal shut-down.

Diuretics alone are ineffective in evacuating fluid accumulated
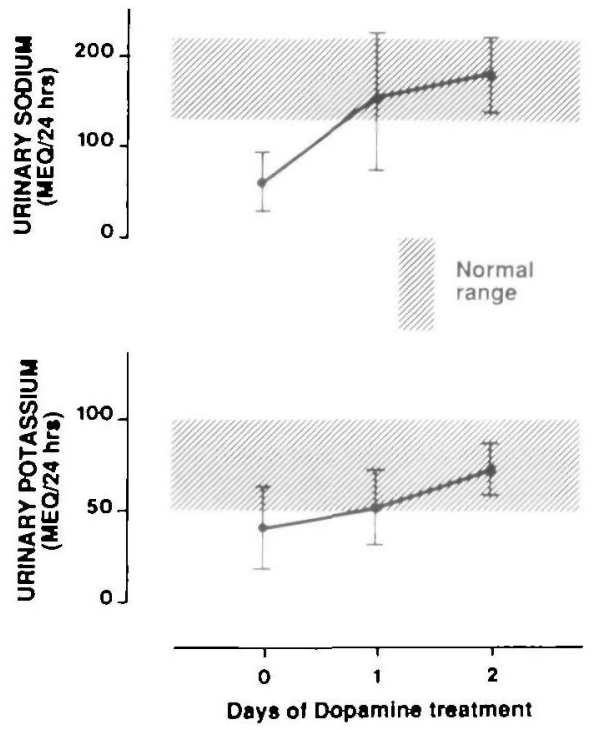

Fig. 2. Variation of urinary excretion of sodium and potassium salts in the first $48 \mathrm{~h}$ of dopamine treatment in seven patients suffering from severe ovarian hyperstimulation syndrome (mean $\pm \mathrm{SD}$ ).

in the 'third space' and as they primarily influence the distal tubules, would have only minimal effects. Indomethacin, as a blocker of prostaglandin biosynthesis, was successfully used for reducing capillary permeability (Knox, 1974; Schenker and Polischuk, 1976) but teratogenic effects are not completely excluded.

Rigid fluid and salt restriction has been demonstrated to be useful in minimizing further ascite formation (Shapiro et al., 1977) but 10 days were necessary before regression of the syndrome began. Moreover, sodium and fluid restriction as the only treatment may induce hypovolaemic shock when the intravascular compartment is already depleted.

In our study, the association of dopamine treatment to increase renal perfusion, and imposition of dietary sodium and proteins to increase serum osmolarity caused fluids to be drawn rapidly from the 'third space' into the intravascular compartment and to be removed through a rise in urine production. This is clearly demonstrated by the negative fluid balance associated in all 
patients with a decrease in weight and abdominal girth. Moreover, intravascular volume is maintained, despite severe fluid restriction and the diuretic effect of dopamine, due to the reabsorption of ascitic fluid.

It is well-known that OHS syndrome may spontaneously regress, but when conception occurs it may also progressively deteriorate in the first weeks of gestation because of endogenous HCG stimulation (Schenker and Polishuk, 1975). The therapeutic approach proposed in this study caused the syndrome to regress rapidly and prevented further complications in all patients, even when there was a high risk that the clinical situation might worsen (multiple pregnancies).

Further studies are needed to confirm these results. In this preliminary study, however, we found the therapeutic administration of low-dosage dopamine to be safe and effective in the management of patients with severe ovarian hyperstimulation.

\section{References}

Felder,R.A., Blecher,M. and Eisner,G.M (1984) Cortical tubular and glomerular dopamine receptors in the rat kidney. Am. J. Physiol., 246, $557-561$.

Gianaroli,L., Ferraretti,A.P., Seracchioli,R., Trounson,A.O , Flamigni,C. and Bovicelli,L. (1986) The successful use of human amniotic fluid for mouse embryo culture and human in vitro fertilization, embryo culture and transfer. Fertil. Steril., 46, 907-913.

Haning, R.U., Strawn,E.V. and Nolten,W.E. (1985) Pathophysiology of the ovarian hyperstimulation syndrome. Obstet. Gynecol., 66, $220-224$.

Kirshan,B., Lee,W., Mauer,M.B. and Cotton,D.B. (1988) Effects of low-dose dopamine therapy in the oliguric patient with preeclampsia. Am. J. Obstet. Gynecol., 159, 604-607.

Knox,G.E. (1974) Antihistamine blockade of the ovarian hyperstimulation syndrome. Am. J. Obstet. Gynecol., 118, 992-994.

Navot,D., Margalioth,E.J., Laufer,N., Bırkenfeld,A., Relou,A., Rosler,A. and Schenker,J.G. (1987) Direct correlation between plasma renin activity and severity of the ovarian hyperstimulation syndrome. Fertil. Steril., 40, 57-61.

Ryley,N.G., Forman,R., Barlow,D., Fleming,K.A. and Trowell,J.M. (1990) Liver abnormality in ovarian hyperstumulation syndrome. Hum. Reprod., 5, 938-943.

Schenker,J.G. and Polishuk,W.Z. (1975) Ovarian hyperstimulation syndrome. Obstet. Gynecol., 46, 23-28.

Schenker,J.G. and Polischuk,W.Z. (1976) Role of prostaglandıns in ovarian hyperstimulation syndrome. Obstet. Gynecol. Surv., 31, $742-745$.

Schenker,J.G. and Weinsteın,D. (1978) Ovarian hyperstimulation syndrome: a current survey. Fertil. Stenl., 30, 255-268.

Shapiro,A.G., Thomas,T. and Epsteın,M. (1977) Management of hyperstimulation syndrome. Fertil. Steril., 28, 237-239.

Younis,J.S., Zeevi,D., Rabinowitz,R., Laufer,N. and Schenker,J.G. (1988) Transient liver function test abnormalities in ovarian hyperstimulation syndrome. Fertil. Steril., 50, 176-178.

Received on June 24, 1991; accepted on October 15, 1991 